\title{
Positive behavioral contrast: Development within sessions'
}

\author{
G. S. REYNOLDS \\ UNIVERSITY OF CALIFORNIA, SAN DIEGO
}

Scattergrams show that the rate of responding to S+ during the formation of a discrimination is not correlated with the rate of responding during the just previous presentation of $S$.

Positive behavioral contrast is habitually observed during the formation of a discrimination between two stimuli. It consists of an increase in the rate of reinforced responding in the presence of the positive stimulus $(\mathrm{S}+)$ when responding is extinguished in the presence of the second stimulus (S-) (Reynolds, 1961a, 1961b). The present paper probes the relationship between the rate of responding in extinction and the magnitude of behavioral contrast by examining the correlation between them during the early stages of the formation of a discrimination. If the prevailing rate of responding during extinction determines the magnitude of behavioral contrast, there should be a substantial negative correlation: the lower the rate of responding in extinction in the presence of $\mathrm{S}-$, the higher the rate of responding in the presence of S+. Method

Subjects. Two pigeons were at $80 \%$ of free-feeding weight.

Apparatus. The chamber contained a key which could be transilluminated with red or green light. Reinforcement was $3.5 \mathrm{sec}$ of access to mixed grain in a feeder.

Procedure. During each daily session, 3 min periods of red illumination of the key were alternated with $3 \mathrm{~min}$ periods of green illumination to a total of thirty presentations of each color. At first, keypecking was maintained in the presence of each of the stimuli on a variable-interval schedule of reinforcement with an average interreinforcement interval of $3 \mathrm{~min}$. After responding had stablized in the presence of each color, and the rates of responding were approximately equal, responding in the presence of the green key was extinguished while the same schedule of reinforcement was continued in the presence of the red key.

Later, the same two Ss were reinforced on a variableratio schedule with an average ratio of 150 until their performances were stable. Then responding was once again extinguished in the presence of the green key while reinforcement continued in the presence of the red key.

\section{Results and Discussion}

Figure 1 shows the median number of responses per min in the presence of each stimulus during the last session before discrimination and during the first two sessions of discrimination. The upper two graphs contain the data obtained for each bird with variableinterval reinforcement, while the lower two graphs contain the data obtained with variable-ratio reinforcement. The notation on the abscissa indicates the schedule of reinforcement associated with each of the two colors.

The birds show the usual decline of responding in extinction (lower curve) as well as positive behavioral contrast, an increase in the rate of responding in the presence of St (upper curve), when responding is extinguished in the presence of the other stimulus.

Figure 2 shows data from a representative bird for variable-interval reinforcement (upper graphs) and for variable-ratio reinforcement (lower graphs). The number of responses per min maintained by reinforcement in the presence of $S+$ is shown as a function of the rate of responding during the immediately preceding presentation of $\mathrm{S}-$. The first graph in each row shows data obtained when responding was rein-
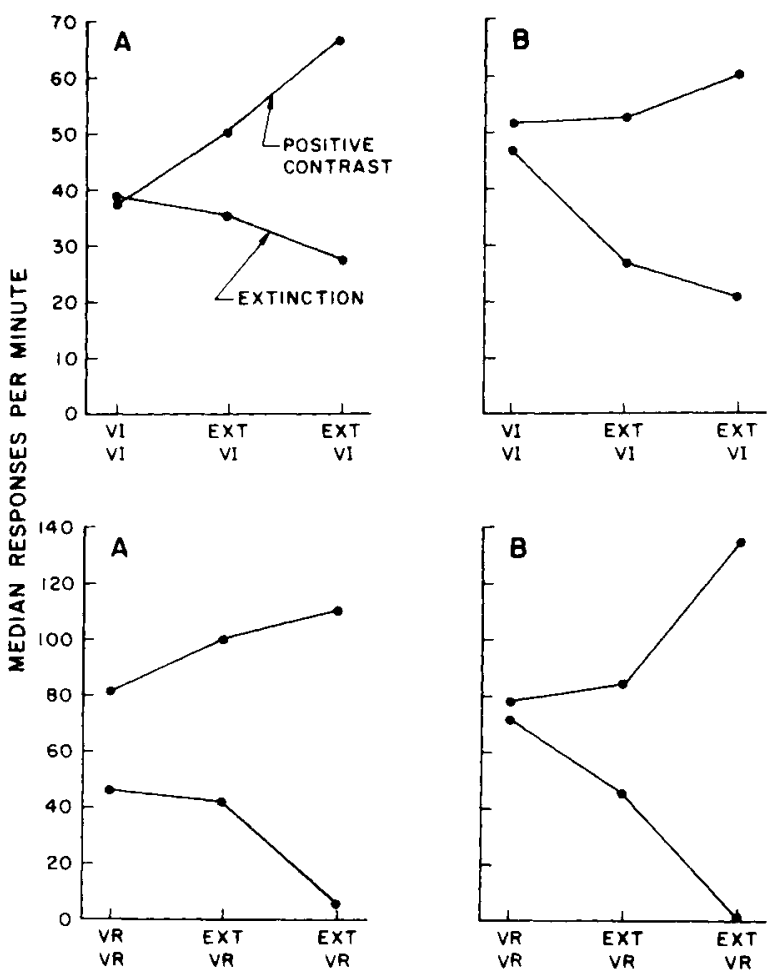

DAILY SESSIONS

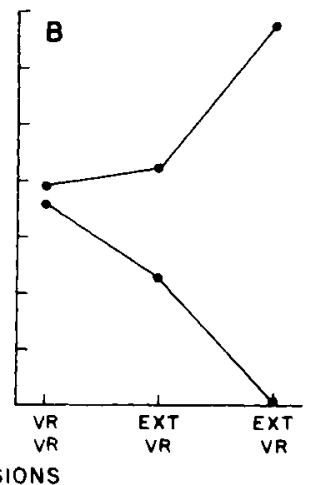

Fig. 1. Median rate of responding by two birds (columns) in each of two components of a multiple schedule for three successive sessions under reinforcement in both components in the first session and extinction in one component in the next session, as indicated on the abscissa. 


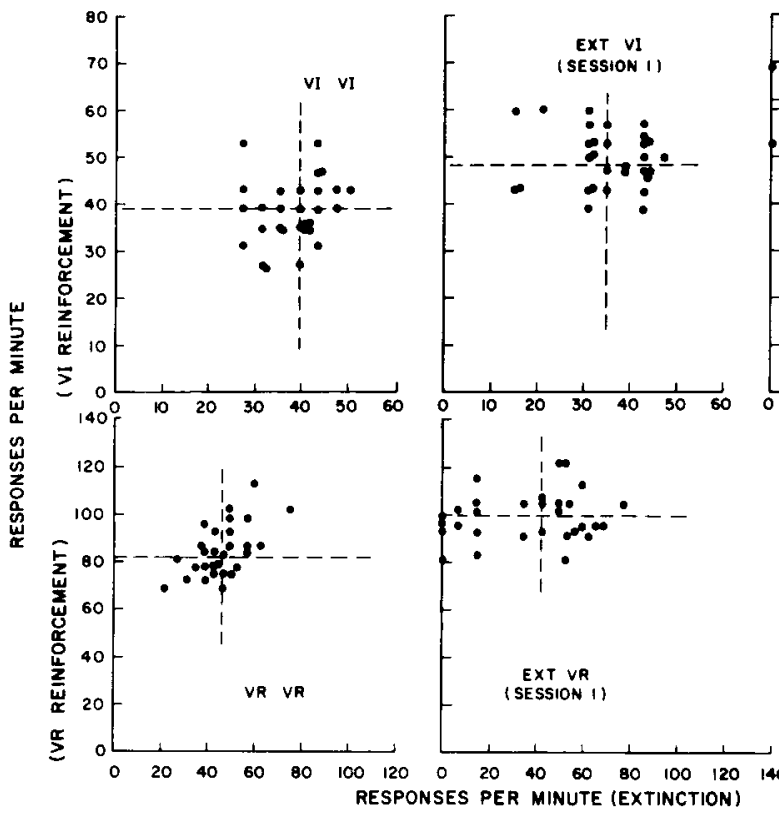

forced in the presence of both stimuli, as indicated by the labels on the graph. The next two graphs show the data obtained from the first and second sessions of discrimination, respectively. The dashed lines on the figures indicate the median ordinate and the median abscissa, plotted in Fig. 1.

What is striking about these data is the lack of negative correlation between the rate of reinforced responding maintained during one stimulus and the rate of responding during extinction in the presence of the other stimulus. Although the rate of reinforced responding increases from session to session (positive behavioral contrast), there is no indication in these data that there is an orderly increase within a session in the rate of reinforced responding as a function of the decreasing rate of responding in extinction.

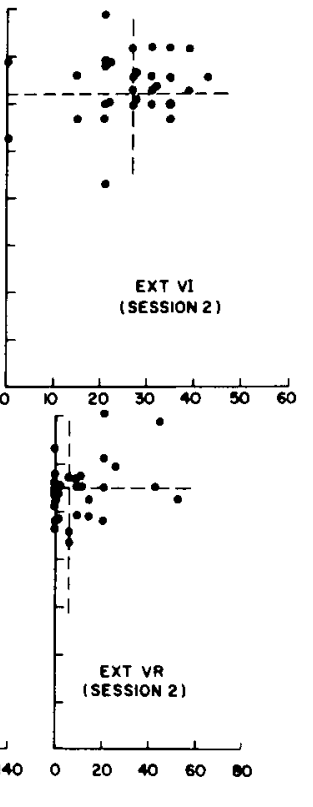

Fig. 2. Scattergrams showing the rate of responding in each $3 \mathrm{~min}$ exposure to reinforcement in one component as a function of the rate of reinforced (column 1) or unreinforced (column 2 and 3) responding in the immediately preceding $3 \mathrm{~min}$ exposure to the second component.
These data by no means rule out an effect on behavioral contrast of responding in extinction. They do, however, make it difficult to account for the development and magnitude of contrast, with either variable-interval or variable-ratio reinforcement, by appealing directly to the prevailing rate of responding in extinction in the presence of $\mathrm{S}-$.

References

REYNOLDS, G. S. Behavioral contrast, J. exp. Anal. Behav., 1961a, 4, 57-71.

REYNOLDS, G. S. An analysis of interactions in a multiple schedule, J. exp. Anal. Behav., 1961b, 4, 107-117.

Note

1. Research supported by NSF Grants GB-2541, GB-5064, and GB-6821. Reprints may be obtained from the author, Department of Psychology, P. O. Box 109, La Jolla, California 92037.

\section{Erratum}

Ferraro, D. P., and Perkins, D. Effects of reinforcement magnitude during successive reinforcementpunishment cycles. Psychon. Sci., 1968,10(5), 183-184. The third paragraph, first sentence (line 22) of the first column of page 184 should be modified to read: "A Friedman analysis of variance on average response rate yielded no significant differences among the four $.06 \mathrm{cc}$ punishment phases or among the four $.02 \mathrm{cc}$ punishment phases which were either one or two cycles removed from the preceeding .06 cc cycle." 